\title{
Trajectory optimization for dynamic couch rotation during volumetric modulated arc radiotherapy
}

\author{
Gregory Smyth ${ }^{1}$, Jeffrey C Bamber ${ }^{1}$, Philip M Evans ${ }^{2}$ and \\ James L Bedford ${ }^{1}$ \\ ${ }^{1}$ Joint Department of Physics, The Institute of Cancer Research and The Royal \\ Marsden NHS Foundation Trust, Sutton, United Kingdom \\ ${ }^{2}$ Centre for Vision, Speech and Signal Processing, University of Surrey, Guildford, \\ United Kingdom \\ E-mail: greg.smyth@icr.ac.uk
}

\begin{abstract}
Non-coplanar radiation beams are often used in three-dimensional conformal and intensity modulated radiotherapy to reduce dose to organs at risk (OAR) by geometric avoidance. In volumetric modulated arc radiotherapy (VMAT) non-coplanar geometries are generally achieved by applying patient couch rotations to single or multiple full or partial arcs. This paper presents a trajectory optimization method for a non-coplanar technique, dynamic couch rotation during VMAT (DCRVMAT), which combines ray tracing with a graph search algorithm. Four clinical test cases (partial breast, brain, prostate only, and prostate and pelvic nodes) were used to evaluate the potential OAR sparing for trajectory optimized DCR-VMAT plans, compared with standard coplanar VMAT. In each case, ray tracing was performed and a cost map reflecting the number of OAR voxels intersected for each potential source position was generated. The least-cost path through the cost map, corresponding to an optimal DCR-VMAT trajectory, was determined using Dijkstra's algorithm. Results show that trajectory optimization can reduce dose to specified OARs for plans otherwise comparable to conventional coplanar VMAT techniques. For the partial breast case, the mean heart dose was reduced by $53 \%$. In the brain case, the maximum lens doses were reduced by $61 \%$ (left) and $77 \%$ (right) and the globes by $37 \%$ (left) and $40 \%$ (right). Bowel mean dose was reduced by $15 \%$ in the prostate only case. For the prostate and pelvic nodes case, the bowel $\mathrm{V}_{50 G y}$ and $\mathrm{V}_{60 G y}$ were reduced by $9 \%$ and $45 \%$ respectively. Future work will involve further development of the algorithm and assessment of its performance over a larger number of cases in site specific cohorts.
\end{abstract}

PACS numbers: $87.55,87.55$. de, $87.53 . \mathrm{Kn}$

Submitted to: Physics in Medicine and Biology 


\section{Introduction}

Volumetric modulated arc radiotherapy (VMAT) combines linear accelerator (linac) gantry rotation during treatment delivery with variation of dose rate, angular speed and aperture shape (Otto 2008). VMAT is increasingly used due to its potential to produce highly conformal dose distributions of similar quality to intensity modulated radiotherapy (IMRT), while reducing treatment fraction duration through efficient delivery (Yu 1999, Yu \& Tang 2011, Teoh et al 2011). In clinical VMAT treatments, the linac gantry arc is generally coplanar with the reconstructed slices of the patient's CT scan. Non-coplanar treatment geometries, often used in three-dimensional conformal radiotherapy (3DCRT) and IMRT to reduce organ at risk (OAR) doses through geometric avoidance, are limited in standard practice to either a static rotation of the patient treatment couch ('couch kick') for a single VMAT arc, or combining multiple full or partial arcs with different static couch angles.

The optimization of non-coplanar beam angles in 3DCRT and IMRT is generally performed manually by experienced treatment planners, although a considerable body of literature exists on automated beam angle optimization approaches (Bortfeld \& Schlegel 1993, Rowbottom et al 1998, 2001, Pugachev et al 2001, Pugachev and Xing 2001, Bangert \& Oelfke 2010, Bangert et al 2012, 2013 and others).

Limited work has been published on the potential for trajectory optimization in rotational therapy, although this may partly reflect limits of conventional delivery technology. Podgorsak et al (1988) proposed combining linac gantry rotation with rotation of the patient couch during stereotactic brain treatment, producing a continuous single gantry arc with dynamic couch rotation such that the non-coplanar trajectory described would not produce opposed beam pairs. This showed promise, improving the dose gradient in normal tissue compared to coplanar arcs. More recently, a number of papers have proposed the use of couch rotation during intensity modulated arc therapy (IMAT) or VMAT treatment for different clinical sites: head and neck (Krayenbuehl et al 2006), prostate and pelvic nodes (Bedford \& Warrington 2010), brain (Yang et al 2011) and breast (Shaitelman et al 2011, Popescu et al 2013). Due to the number of different terms in the literature, and to distinguish between other possible motions where trajectories can be optimized (e.g. rotation of the beam collimator), in this paper we refer to dynamic couch rotation during VMAT as DCR-VMAT.

Although these papers evaluate the potential of dynamic couch rotation for the respective clinical sites, the common limitation is a lack of direct trajectory optimization. With the exception of Yang et al (2011), all trajectories were user-defined and therefore notionally 'arbitrary'. Yang et al identified optimal individual source positions using a beam's eye view volumetrics technique (Chen et al 1992, Myrianthopoulos et al 1992, McShan et al 1995). Partial-arc trajectories were not directly optimized but determined by forming clusters of these source positions, which were then smoothed and extended. Mizowaki et al (2013) also described a technique similar to DCR-VMAT - "3D unicursal irradiation" - for the Vero gimballed linac (Vero4DRT, Mitsubishi Heavy Industries Ltd, 
Tokyo), which involved rotation of the linac's O-ring mounting about the vertical axis. However, trajectories used for the 'unicursal' plans were not optimized.

This paper proposes a trajectory optimization technique - strictly a trajectory customization heuristic (Bangert 2011) - for single-arc DCR-VMAT that determines a minimum-cost trajectory through a cost map using a graph search algorithm. Customization of the cost map to reflect a range of cost functions is possible. In this paper, however, a simple but effective cost function based on ray tracing calculations of OAR voxel intersections was implemented. The algorithm will be presented with examples demonstrating applicability to a broad range of clinical sites. Plans for future development and refinement of the technique are also discussed.

\section{Materials and methods}

\subsection{Trajectory optimization}

A trajectory optimization method was implemented in MATLAB (v2010b, The Mathworks, Nantick, MA). The method is outlined here, with more detail given below. For each case under investigation, a Digital Imaging and Communications in Medicine (DICOM) radiotherapy structure set was read into the trajectory optimizer and ray tracing through the dataset was performed for all permitted source positions. A cost for each source position was determined from ray-OAR voxel intersections, producing a cost map. A graph search algorithm was used to find a path through the map, corresponding to a VMAT trajectory with minimum total cost. The trajectory was then exported to an in-house treatment planning system (AutoBeam v5.2a) for VMAT plan optimization (Bedford 2009). The VMAT plan was exported to Pinnacle ${ }^{3}$ (v9.2, Philips Radiation Oncology Systems, Fitchburg, WI) and a final dose calculation was performed.

Initial testing of the trajectory optimization method was performed using a geometric phantom containing OARs designed to produce known DCR-VMAT trajectories, with either a 'simple' static couch angle or 'complex' dynamic couch rotation.

2.1.1. Data input. The trajectory optimization method required a DICOM radiotherapy structure set, containing isocentre position, external contour, planning target volume (PTV), and OARs. Values for gantry start and stop positions and the calculation resolution, or control point spacing, of gantry and couch rotation were also used.

2.1.2. Ray tracing and cost map generation. The trajectory optimization method calculated source positions for permitted gantry and couch angle combinations and performed ray tracing using a published algorithm (Siddon 1985). 
The patient dataset was divided into equal sized voxels $(2.5 \times 2.5 \times 2.5 \mathrm{~mm})$, with intersection calculations simplified by relating the voxel boundaries to equispaced parallel planes in three dimensions. The intersection of a ray with the first plane in each dimension was calculated and, by parametrizing the ray, intersections with all subsequent planes could be inferred. A list of intersected voxels, identified by indices in the three dimensions, was then produced.

In order to improve the efficiency of ray tracing, the number of rays cast was reduced according to the following scheme. The centre coordinates of PTV voxels were transformed onto the beam's eye view (BEV) plane at the isocentre for each gantry and couch angle combination, according to Siddon (1981). The BEV plane was divided into a grid of beam elements (bixels) with resolution $2.5 \times 2.5 \mathrm{~mm}$. Where a PTV voxel centre transformed onto the BEV plane lay within a bixel, that bixel was added to the list of beamlets to be traced. Ray tracing was performed along the central axis of each identified beamlet.

Couch rotations between $90^{\circ}$ and $270^{\circ}$ (International Electrotechnical Commission (IEC) 61217 standard) were eliminated due to physical restriction of the Elekta Synergy linac (Elekta AB, Stockholm, Sweden) used. Collision avoidance was implemented by defining a range of explicitly forbidden couch and gantry combinations determined from measurements on the linac. Potential collision regions were defined for gantry angles between $30^{\circ}$ and $330^{\circ}$, with less restriction for brain cases where vertex orientations were permitted.

For a specific combination of couch angle $c$ and gantry angle $g$, the associated cost $C$ is given by the total number of OAR voxels traversed by the rays cast:

$$
C_{c, g}=\sum_{r \in R} \sum_{o \in O} \sum_{i \in o} n_{i}
$$

where $R$ is the total number of rays $r$ traced, $O$ is the set of all organs at risk $O$ being avoided, and $n_{i}$ denotes the intersection of voxel $i$ by ray $r$.

The costs for all permitted combinations of couch and gantry angle were assigned to a matrix and displayed as a cost map, showing the relative volume of OAR irradiated from each source position.

2.1.3. Trajectory determination. Consider a geometric graph $G=[V, E]$ defined by a number of vertices, $V$, connected by a number of edges, $E$, with defined penalties known as edge weights. In a travelling salesman problem, each vertex might represent a city with edge weights representing geographical distances between them. A graph search algorithm can be used to determine a minimum-cost path from one vertex, or city, to any other (Cormen et al 2009). Applied to the VMAT trajectory problem in this paper, each vertex could represent a source position, defined by gantry and couch rotations, with the edge weight when moving from an adjacent source position to source position $(c, g)$ defined by cost map element $C_{c, g}$. Edge connections within the graph for a given source position were defined as each of the immediately adjacent elements in the cost map, subject to physical or collision limits. 
Dijkstra's algorithm (Dijkstra 1959) is a graph search technique which determines the minimum distance between two vertices by progressively visiting adjacent vertices and updating a list of path costs (Cormen et al 2009). An implementation of Dijkstra's algorithm $\dagger$ was used to determine the minimum-cost path between all combinations of permitted trajectory start and end positions, producing a matrix of minimum-cost paths. However, as only one minimum-cost path is returned for each combination of start and end vertices, the existence of additional paths with minimum cost is not precluded.

To reduce the likelihood of cost maps with zero-penalty regions producing trajectories with unnecessary additional path steps, the cost of a single voxel intersection was added to all elements within the cost matrix. The overall minimum-cost for all combinations of start and end position was found.

The cost of trajectory $t \in T$ is then given by:

$$
C_{t}=\sum_{p \in P} C_{c, g}
$$

where $p$ is the number of steps in the path $P$.

The optimal trajectory is then given by:

$$
\min C_{t} \forall t \in T
$$

For cost maps which result in multiple trajectories of equal cost, these were compared to find the most efficient path in terms of number of control points required. The total cost of the resulting 'complex' DCR-VMAT trajectory was then compared with the costs of all 'simple' static couch angle trajectories. If one of the 'simple' trajectories had an equal cost to the 'complex' trajectory, the 'simple' trajectory was preferred.

2.1.4. Trajectory import and planning. The steps comprising the final minimumcost path were converted to couch and gantry angles, corresponding to the DCRVMAT trajectory control points. The trajectory was exported to AutoBeam for plan optimization and final dose calculation in Pinnacle ${ }^{3}$.

Optimization in AutoBeam followed by final calculation in Pinnacle ${ }^{3}$ is standard practice for AutoBeam plans to ensure accurate dose calculation. While DCR-VMAT trajectories can be imported into Pinnacle ${ }^{3}$, it is not currently possible to produce DCRVMAT plans of acceptable quality using Pinnacle ${ }^{3}$ alone.

\subsection{Clinical cases}

In order to evaluate any clinical benefit of trajectory optimization, four cases were planned using both coplanar VMAT and DCR-VMAT. The sites investigated were: partial breast, brain, prostate only, and prostate and pelvic nodes, chosen to provide a broad range of sites and be comparable with the existing DCR-VMAT literature. Treatment planning was performed using a nominal $6 \mathrm{MV}$ photon beam from an Elekta

$\dagger$ Kirk J Advanced Dijkstra's Minimum Path Algorithm. http://www.mathworks.com/matlabcentral/ fileexchange/20025-advanced-dijkstras-minimum-path-algorithm Last accessed 13 June 2013 
Synergy linac with a multileaf collimator (MLC) projected leaf width at the isocentre of $4 \mathrm{~mm}$ (Beam Modulator, Elekta AB), with the exception of the prostate and pelvic node case which was planned using a $10 \mathrm{~mm}$ MLC leaf width at the isocentre (MLCi, Elekta $\mathrm{AB})$ due to the small maximum field size of the Beam Modulator treatment head.

To ensure any difference between plans was due to the different trajectories, the same AutoBeam parameters, optimization objectives and objective weights were used for both VMAT and DCR-VMAT plans for a given site. Final dose calculation for all plans was performed in Pinnacle ${ }^{3}$ using the collapsed cone convolution algorithm on a $2.5 \times 2.5 \times 2.5 \mathrm{~mm}$ dose grid, with the exception of the brain case which was calculated on a $2 \times 2 \times 2 \mathrm{~mm}$ dose grid due to the small size of its PTV and OARs.

In most cases, a single OAR was chosen for trajectory optimization, having considered its clinical significance and the potential sparing from non-coplanar geometries. An example of using multiple OARs during trajectory optimization is presented as part of the brain case. Determining trajectories using multiple OARs, or OARs substantially overlapping the PTV, requires conflicting clinical priorities to be reflected within the trajectory optimization algorithm. Different methods for this, such as the use of relative importance weighting factors, will be investigated as the algorithm is developed during site-specific clinical investigations of the DCR-VMAT technique. Trajectory optimization using a limited number of OARs was felt to be sufficient for the more general investigation of the algorithm presented in this paper.

2.2.1. Partial breast. The partial breast case was a right-sided tumour bed sequential boost from the Intensity Modulated and Partial Organ Radiotherapy (IMPORT High) Phase III clinical trial (Donovan et al 2011), prescribed to a PTV mean dose of 16 Gy in 8 fractions. Trajectory optimization was used to determine a partial-arc DCRVMAT trajectory, with gantry and couch spacing of $2^{\circ}$ per control point, between the gantry angle limits of the original whole breast tangent fields $\left(48^{\circ}\right.$ and $\left.224^{\circ}\right)$ minimizing the volume of heart irradiated. The DCR-VMAT plan was compared with a coplanar partial-arc VMAT plan, with both plans produced with nominal gantry speed of $3.0^{\circ} / \mathrm{s}$ and MLC leaves allowed to move within an envelope specified as the PTV plus penumbra margin of $6 \mathrm{~mm}$ anteroposteriorly and laterally, and $10 \mathrm{~mm}$ superoinferiorly.

2.2.2. Brain. The brain case was an intrasuprasellar craniopharyngioma, prescribed to a PTV mean dose of 54 Gy in 30 fractions. OARs were the optic chiasm, optic nerves, globes, lenses, cochleae and brainstem, of which the bilateral globes and lenses were used in trajectory optimization, with gantry and couch spacing of $2^{\circ}$ per control point. VMAT and DCR-VMAT treatment planning was performed for a single anticlockwise arc between gantry $179^{\circ}$ and $181^{\circ}$ with nominal gantry speed of $3.0^{\circ} / \mathrm{s}$ and MLC leaves conforming to an envelope specified as the PTV plus penumbra margin of 4 $\mathrm{mm}$ anteroposteriorly and laterally, and $5 \mathrm{~mm}$ superoinferiorly.

Optimal trajectories for two anticlockwise partial-arcs were also determined for the same parameters; the first between gantry $179^{\circ}$ and $7^{\circ}$ and the second between gantry 
Table 1. Beam orientations for the non-coplanar 3D conformal brain plan.

\begin{tabular}{clccc}
\hline \multirow{2}{*}{ Beam } & Orientation & Energy $(\mathrm{MV})$ & Angle $\left(^{\circ}\right)$ \\
& Gantry & Couch \\
\hline 1 & Superior anterior oblique & 6 & 45 & 270 \\
2 & Inferior right oblique & 6 & 270 & 345 \\
3 & Inferior left oblique & 6 & 90 & 15 \\
4 & Superior right anterior oblique & 6 & 290 & 15 \\
5 & Superior left anterior oblique & 6 & 70 & 345 \\
6 & Superior posterior oblique & 10 & 115 & 270 \\
\hline
\end{tabular}

$7^{\circ}$ and $181^{\circ}$. A partial-arc DCR-VMAT (pDCR-VMAT) plan was created using the same optimization objectives and settings as for the single-arc.

A second full single-arc DCR-VMAT trajectory was determined using multiple OARs - bilateral globes, lenses, optic nerves, cochleae and the optic chiasm - for optimization. Planning was performed for this trajectory using the same optimization objectives and settings as for original single-arc DCR-VMAT plan.

A six-field 3DCRT plan was produced to compare the potential OAR sparing of a conventional non-coplanar technique with the various VMAT plans; beam orientations are summarized in Table 1. A conformal technique was preferred over IMRT due to the small size of the PTV and for consistency with the conformal VMAT arcs used in this case.

2.2.3. Prostate only. The prostate only case was from the conventional linac-based IMRT arm of the PACE Phase III clinical trial $\ddagger$, prescribed to a PTV mean dose of 78 Gy in 39 fractions (Tree et al 2013). OARs were the bladder, rectum, proximal section of bowel and femoral heads, of which the bowel was used in trajectory optimization with gantry and couch spacing of $4^{\circ}$ per control point. A control point spacing of $4^{\circ}$ was used due to the relatively simple volume shape, consistent with local coplanar VMAT practice. VMAT and DCR-VMAT treatment planning was performed for a single anticlockwise arc between gantry $178^{\circ}$ and $182^{\circ}$ with nominal gantry speed of $3.0^{\circ} \mathrm{s}$ and MLC leaves allowed to move within an envelope specified as the PTV plus penumbra margin of $6 \mathrm{~mm}$ anteroposteriorly and laterally, and $10 \mathrm{~mm}$ superoinferiorly.

The dosimetric impact of systematic couch misalignment during DCR-VMAT was investigated for this case. The couch angles of each control point in the DCR-VMAT plan were modified by $\pm 1^{\circ}$ and $\pm 2^{\circ}$ and the dose was recalculated using the original monitor units to simulate treatment error.

$\ddagger$ Prostate Advances in Comparative Evidence (PACE)

http://www.clinicaltrials.gov/ct2/show/NCT01584258 Last accessed 9 July 2013 
2.2.4. Prostate and pelvic nodes. The prostate and pelvic nodes (PPN) case was from the PIVOTAL Phase II clinical trial $\S$. Doses of 74 Gy, 71 Gy and 60 Gy were planned in a simultaneous integrated boost (SIB) technique to four PTVs: PTV74 (prostate grown to exclude the rectum with a $5 \mathrm{~mm}$ margin in all dimensions, except posteriorly where no margin was applied), PTV71 (PTV74 plus $5 \mathrm{~mm}$ isotropic margin), PTV60S (combined prostate and seminal vesicles plus $10 \mathrm{~mm}$ isotropic margin) and PTV60N (pelvic nodes plus $5 \mathrm{~mm}$ isotropic margin). Plans were prescribed to a PTV74 mean dose of $74 \mathrm{~Gy}$ in 37 fractions. All PTVs were combined into a single target volume for trajectory optimization. OARs were the bladder, rectum, bowel and femoral heads, of which bowel was used in the trajectory optimization, with gantry and couch spacing of $2^{\circ}$ per control point.

VMAT and DCR-VMAT treatment planning was performed with nominal gantry speed of $1.5^{\circ} / \mathrm{s}$ and $10 \mathrm{~mm}$ MLC leaves allowed to move within an envelope specified as the PTV plus penumbra margin of $6 \mathrm{~mm}$ anteroposteriorly and laterally, and $11 \mathrm{~mm}$ superoinferiorly. Three VMAT arcs were used for treatment planning - two anticlockwise arcs from gantry $179^{\circ}$ to $181^{\circ}$, and one clockwise from $181^{\circ}$ to $179^{\circ}$. Trajectory optimization was performed once for this case, with the separate VMAT arcs used in planning passing back and forth over the same trajectory. Optimization objective values for both VMAT and DCR-VMAT plans were modified from Bedford (2013).

\section{Results}

\subsection{Partial breast}

Trajectory optimization produced a partial-arc with a static couch angle of $346^{\circ}$ during gantry rotation, rotated to minimise the volume of heart irradiated. This is shown in Figure 1(a), with the 'hot' region indicating source positions with higher numbers of OAR voxels intersected during ray tracing. As described in 2.1.3, trajectory optimization was designed to prefer a 'simple' static couch angle trajectory over 'complex' dynamic couch trajectories with equal total cost. Coronal sections of the VMAT and DCR-VMAT plans are presented in Figure 2; note the rotation of the 1 and 2 Gy isodose lines away from the heart in the DCR-VMAT plan. Dose volume histograms (DVHs) are presented in Figure 3(a) and dose statistics are presented in Table 2.

While the heart maximum dose was reduced by $4 \%$ in the DCR-VMAT plan, mean heart dose was reduced by $53 \%$ with the reduction clearly shown in Figure 3(a). Doses to other OARs were either reduced by DCR-VMAT or comparable, with contralateral breast maximum dose reduced by $23 \%$ and mean dose reduced by $70 \%$, contralateral

$\S$ Prostate and pelvis versus prostate alone treatment for locally advanced prostate cancer: A randomised phase II trial of prostate and pelvis versus prostate-alone IMRT for locally advanced prostate cancer.

http://rttrialsqa.dnsalias.org/Pivotal/PIVOTAL\%20trial\%20website\%20summary.htm Last accessed 9 July 2013 


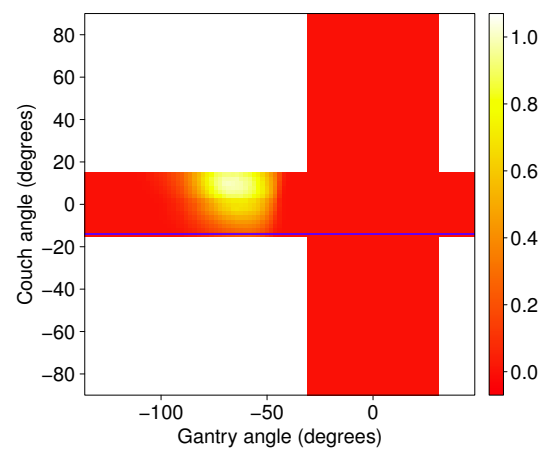

(a) Partial breast

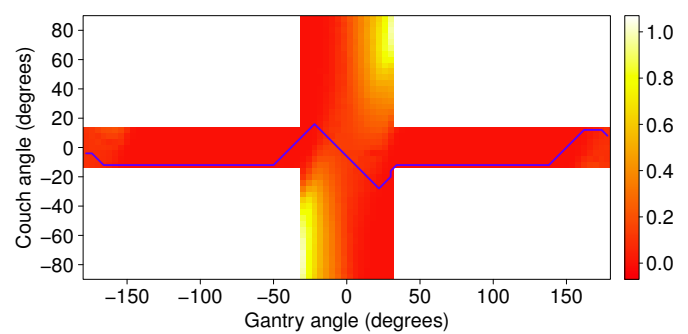

(c) Prostate only

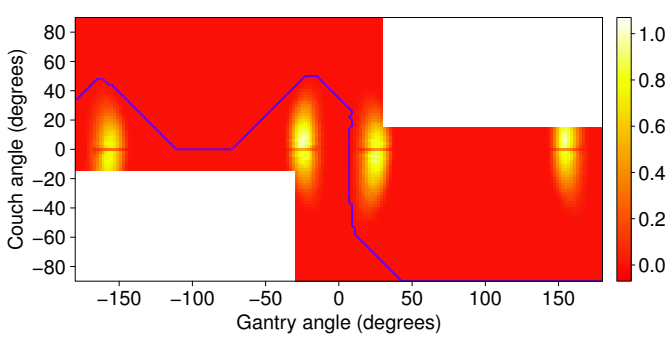

(b) Brain

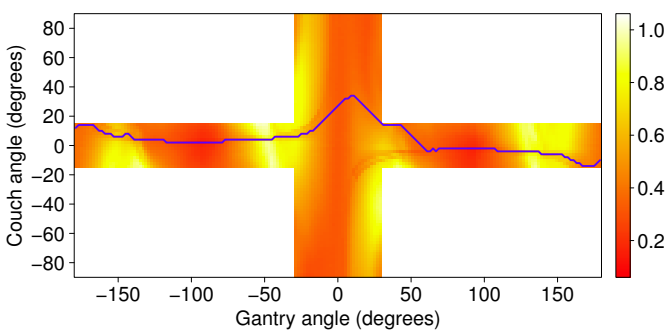

(d) Prostate and pelvic nodes

Figure 1. Trajectory optimization results for (a) partial breast, (b) brain, (c) prostate only, and (d) prostate and pelvic nodes cases. Cost maps are displayed as a heat map, normalized to the highest overall cost. Red regions indicate low cost and yellow regions indicate high cost; white regions are forbidden due to potential collisions. The DCRVMAT trajectories are overlaid as a solid blue line, starting from the right hand side of each graph for the anticlockwise arc. (Note scales are displayed to correctly show the continuous trajectory search space; angles within the range $-179^{\circ}$ and $-1^{\circ}$ on the cost map correspond to those between $181^{\circ}$ and $359^{\circ}$ in the International Electrotechnical Commission (IEC) 61217 standard.)

lung maximum dose reduced by $14 \%$ and mean dose reduced by $16 \%$. Ipsilateral lung maximum dose was reduced by $3 \%$, while mean dose was unchanged; ipsilateral breast excluding PTV mean dose increased by $7 \%$.

\subsection{Brain}

3.2.1. DCR-VMAT comparison. The trajectory optimization result is shown in Figure 1(b), and included an initial vertex section of arc. Axial sections through the VMAT and DCR-VMAT distributions are shown in Figure 2; note how the 8 Gy isodose avoids the lenses in the DCR-VMAT plan. Dose volume histograms are presented in Figure 3(b), with dose statistics presented in Table 3 as VMAT and DCR1 respectively. Maximum doses to the left lens, right lens, left globe, right globe, left cochlea and right cochlea were considerably reduced: by $61 \%, 77 \%, 37 \%, 40 \%, 53 \%$ and $53 \%$ respectively. Maximum doses for other OARs were comparable, with no change in the left optic nerve, a $1 \%$ 


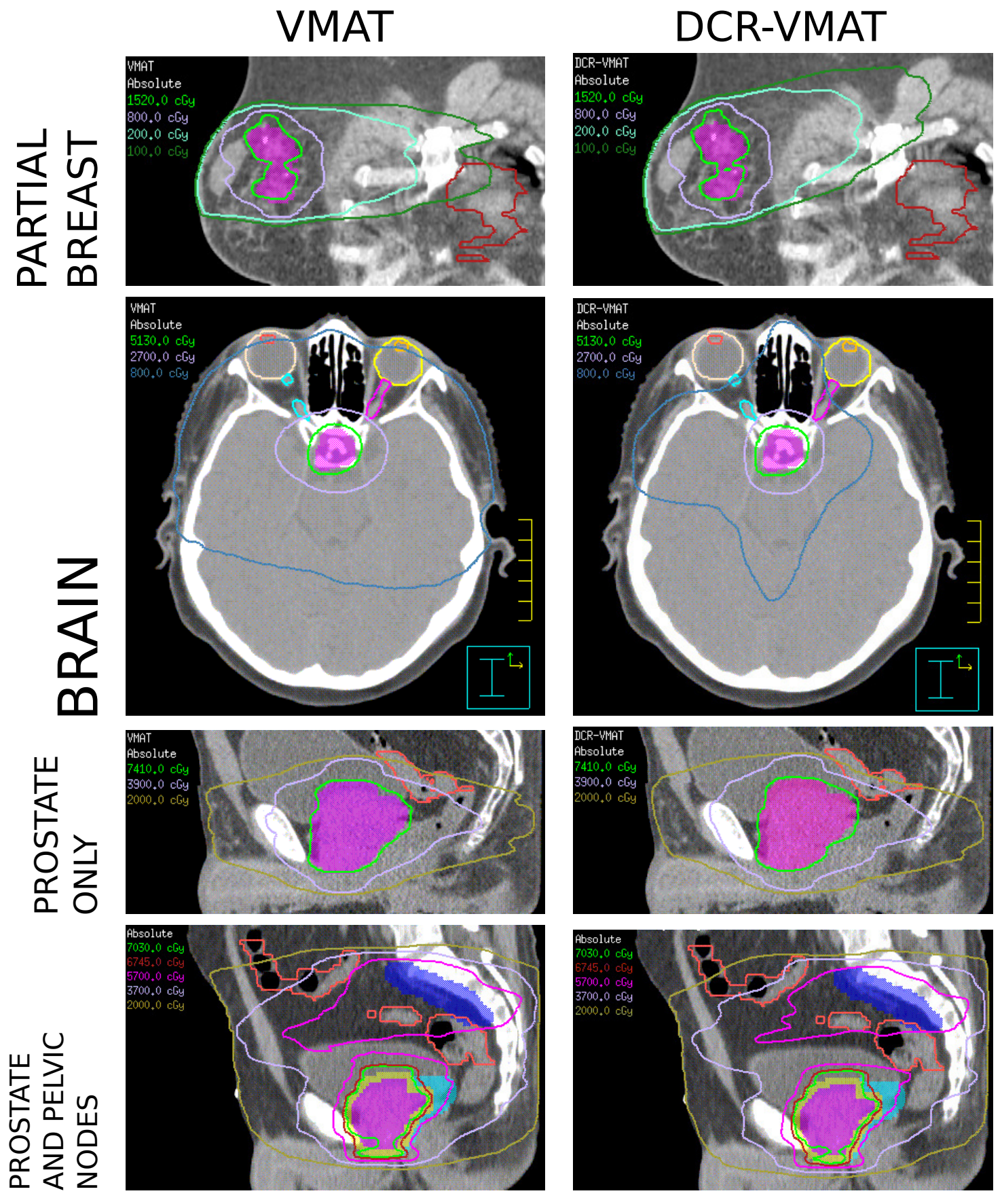

Figure 2. From top: coronal views of the partial breast plans, axial views of the brain plans, sagittal views of the prostate only plans, and sagittal views of the prostate and pelvic nodes plans. PTVs are displayed in colourwash, with OARs of interest shown as contours. Isodose lines are displayed corresponding to the $95 \%$ dose of each PTV, $50 \%$ dose level and other illustrative dose levels. Isodoses shown are: $15.2 \mathrm{~Gy}, 8 \mathrm{~Gy}, 2$ Gy and 1 Gy for the partial breast; $51.3 \mathrm{~Gy}, 27 \mathrm{~Gy}$ and $8 \mathrm{~Gy}$ for the brain; $74.1 \mathrm{~Gy}$, 39 Gy and 20 Gy for the prostate only; 70.3 Gy, 67.45 Gy, 57 Gy, 37 Gy and 20 Gy for the prostate and pelvic nodes.

increase for the right optic nerve, and $2 \%$ reduction for the brainstem.

The maximum dose to the optic chiasm increased by $2 \%$ for DCR-VMAT. Both 


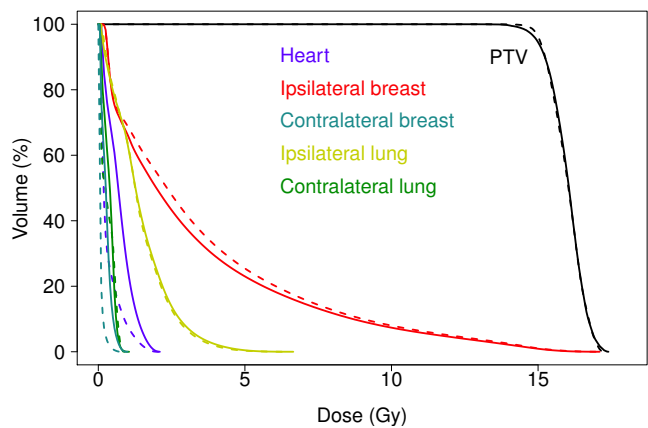

(a) Partial breast



(c) Prostate only

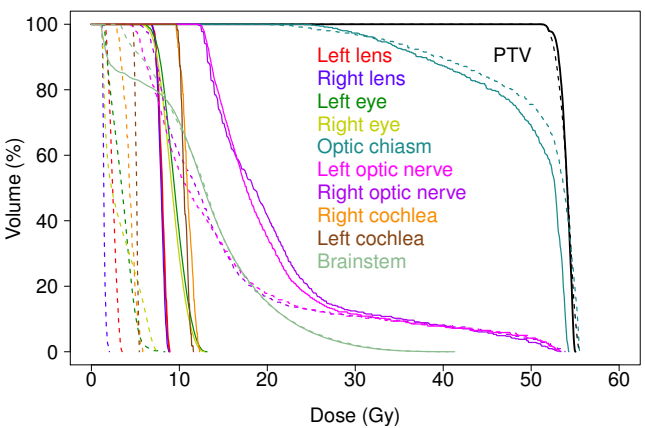

(b) Brain

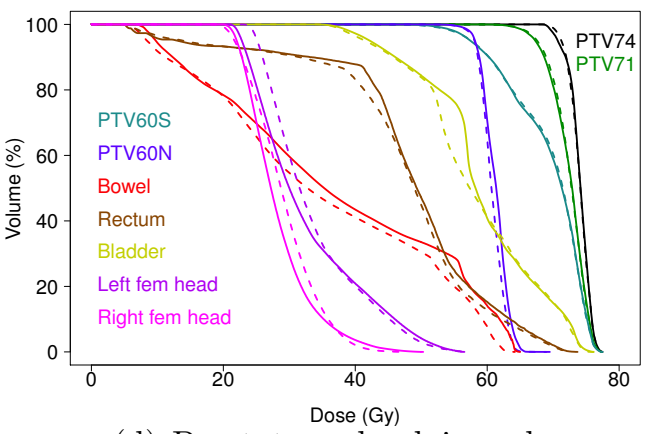

(d) Prostate and pelvic nodes

Figure 3. Dose volume histograms, showing VMAT (solid line) against DCR-VMAT (dashed), for (a) partial breast, (b) brain, (c) prostate only, and (d) prostate and pelvic nodes cases.

Table 2. Organ at risk dose statistics for the partial breast case.

\begin{tabular}{llcc}
\hline \multirow{2}{*}{ Organ at risk } & & \multicolumn{2}{c}{ Dose (cGy) } \\
& Criterion & VMAT & DCR-VMAT \\
\hline Ispilateral breast excluding PTV & Maximum dose & 1708 & 1716 \\
& Mean dose & 332 & 356 \\
Ipsilateral lung & Maximum dose & 660 & 640 \\
Heart & Mean dose & 140 & 140 \\
& Maximum dose & 209 & 201 \\
Contralateral breast & Mean dose & 72 & 34 \\
& Maximum dose & 97 & 75 \\
Contralateral lung & Mean dose & 27 & 8 \\
& Maximum dose & 102 & 88 \\
& Mean dose & 37 & 31 \\
\hline
\end{tabular}


Table 3. Organ at risk dose statistics for all brain case plans.

\begin{tabular}{lccccc}
\hline & \multicolumn{5}{c}{ Maximum dose (Gy) } \\
Organ at risk & VMAT & DCR1 & pDCR1 & DCR2 & 3DCRT \\
\hline Left lens & 8.9 & 3.5 & 1.9 & 4.8 & 1.3 \\
Right lens & 8.8 & 2.0 & 1.5 & 4.8 & 1.2 \\
Left globe & 13.1 & 8.3 & 5.0 & 10.6 & 2.1 \\
Right globe & 12.8 & 7.7 & 7.6 & 10.7 & 1.8 \\
Left optic nerve & 53.2 & 53.2 & 53.5 & 53.8 & 54.4 \\
Right optic nerve & 53.4 & 53.8 & 54.5 & 54.0 & 53.4 \\
Optic chiasm & 54.3 & 55.5 & 56.0 & 54.4 & 55.7 \\
Left cochlea & 11.6 & 5.4 & 2.1 & 5.2 & 7.6 \\
Right cochlea & 12.3 & 5.8 & 1.6 & 7.5 & 7.9 \\
Brainstem & 41.3 & 40.5 & 43.5 & 42.0 & 32.5 \\
\hline
\end{tabular}

Table 4. Conformity statistics for the brain case.

\begin{tabular}{lccccc}
\hline \multirow{2}{*}{ Criterion } & VMAT & DCR1 & pDCR1 & DCR2 & 3DCRT \\
\hline $\mathrm{V}_{95 \%}$ & 9.6 & 9.8 & 9.6 & 10.0 & 9.5 \\
$\mathrm{~V}_{50 \%}$ & 42.3 & 37.0 & 39.6 & 41.1 & 44.2 \\
$\mathrm{~V}_{8 G y}$ & 444.3 & 327.8 & 330.1 & 374.1 & 411.5 \\
\hline $\mathrm{V}_{x}=$ volume receiving a dose of $x$ Gy or $\%$.
\end{tabular}

VMAT and DCR-VMAT plans breached local optimal dose constraints for optic chiasm (maximum dose less than $54 \mathrm{~Gy}$ ), however, in this case $46.4 \%$ of the OAR lay within the PTV which was prescribed a mean dose of $54 \mathrm{~Gy}$. In practice, the clinical treatment plan chosen for this patient compromised PTV dose to keep optic chiasm dose below 54 Gy. It was felt preferable to maintain PTV coverage in this comparison to evaluate the effect of trajectory optimization alone.

3.2.2. Dual partial-arc DCR-VMAT. The pDCR-VMAT trajectory consisted of two partial-arcs with static couch angles of $270^{\circ}$ and $50^{\circ}$ respectively. Dose statistics are presented as pDCR1 in Table 3. The plan showed further improvement over single-arc DCR-VMAT (DCR1) in sparing the bilateral lenses, globes and cochleae, although bilateral optic nerves, optic chiasm and brainstem maximum doses were modestly increased.

By creating two partial-arcs the 'linking' section of the DCR-VMAT trajectory seen in Figure 1(b), where the couch rotated during limited gantry rotation, was eliminated. This may have contributed to the improvements in OAR sparing by reducing the section of arc directed between the globes.

3.2.3. Trajectory optimization using multiple OARs. Dose statistics for multiple OAR single-arc trajectory optimization are presented as DCR2 in Table 3. Compared with 
the original coplanar VMAT plan, DCR2 reduced the maximum dose to the lenses by $46 \%$ (left) and $45 \%$ (right), globes by 19\% (left) and 16\% (right), and cochleae by $55 \%$ (left) and $39 \%$ (right). With the exception of the left cochlea, improvements were not as great as those for the DCR1 plan. Bilateral optic nerve doses were broadly similar in each of the three plans, although the brainstem dose increased in DCR2. The optic chiasm maximum dose was essentially unchanged from the coplanar VMAT plan.

By incorporating multiple OARs that may be best spared from competing couch and gantry combinations into the trajectory optimization, the overall sparing effect may be reduced. Incorporating indications of clinical priority, or "importance factors", into the trajectory optimization may enable better targeting of improvements at preferred OARs while simultaneously taking account of multiple OARs.

3.2.4. Comparison with non-coplanar 3DCRT. Dose statistics for the non-coplanar 3DCRT plan are presented in Table 3. Bilateral globes and lenses were best spared by 3DCRT due to judicious choice of beam angles, however pDCR-VMAT produced a similar level of lens sparing while additionally sparing the bilateral cochleae. All DCR-VMAT techniques demonstrated improved sparing for the cochleae over 3DCRT, although this was small for the right cochlea in DCR2. Brainstem maximum dose was also best spared in 3DCRT, however neither of the trajectory optimization approaches attempted to avoid it. Optic chiasm dose was improved for all single-arc VMATs, however the optic chiasm significantly overlapped the PTV and therefore its maximum dose likely reflects deviations in PTV uniformity. Volumes of 27 Gy (50\% isodose) and 8 Gy were improved for all DCR-VMAT approaches compared with 3DCRT as shown in Table 4.

\subsection{Prostate only}

3.3.1. DCR-VMAT comparison. Trajectory optimization results are shown in Figure 1(c). Sagittal views of VMAT and DCR-VMAT plans are shown in Figure 2; note the reduction in bowel encompassed by the 39 Gy and 20 Gy isodoses in the DCR-VMAT plan. Dose volume histograms for the PTV, bowel, bladder and rectum are shown in Figure 3(c), while dose statistics are presented in Table 5.

While the bowel maximum dose increased by $1 \%$ with DCR-VMAT, the mean dose decreased by $15 \%$. Due to the geometry of the prostate and OARs, improvement in some OAR DVHs is likely to come at the expense of others and this is shown in mixed results for the rectum, bladder and femoral heads. Rectum maximum dose was reduced by $1 \%$ but mean dose increased by $2 \%$; bladder maximum dose reduced by $1 \%$, while mean dose increased by $5 \%$. Femoral head mean doses reduced by $28 \%$ for the right femoral head but increased by $15 \%$ for the left femoral head.

Both the rectum and bladder overlapped the PTV, which was prescribed a mean dose of $78 \mathrm{~Gy}$. While no maximum dose constraints were stated in the PACE trial protocol for these OARs, rectum V75Gy and bladder V74Gy were required to be less 
Table 5. Organ at risk dose statistics for the prostate only case.

\begin{tabular}{llcc}
\hline \multirow{2}{*}{ Organ at risk } & Criterion & \multicolumn{2}{c}{ Dose (Gy) } \\
\hline Rectum & Maximum dose & 81.0 & 79.8 \\
& Mean dose & 48.4 & 49.3 \\
\multirow{2}{*}{ Bladder } & Maximum dose & 81.3 & 80.6 \\
\multirow{2}{*}{ Bowel } & Mean dose & 28.6 & 29.9 \\
\multirow{2}{*}{ Left femoral head } & Maximum dose & 65.9 & 66.8 \\
Right femoral head & Mean dose & 23.0 & 19.5 \\
\hline
\end{tabular}

than $15 \%$. These constraints were met by both VMAT and DCR-VMAT plans, as seen in Figure 3(c).

3.3.2. Effect of couch rotation errors. For systematic errors in couch rotation of up to $\pm 2^{\circ}$, differences in PTV, rectum and bladder maximum and mean dose, bowel maximum dose and left femoral head mean dose were within $\pm 1 \%$ of the DCR-VMAT plan; bowel mean doses were less than $\pm 1.5 \%$ for $\pm 1^{\circ}$ and $\pm 3 \%$ for $\pm 2^{\circ}$. The greatest dosimetric effect was seen in the right femoral head, with errors of almost $\pm 5 \%$ for $\pm 1^{\circ}$ and $\pm 10 \%$ for $\pm 2^{\circ}$.

\subsection{Prostate and pelvic nodes}

Trajectory optimization results are shown in Figure 1(d). Sagittal sections for both plans are shown in Figure 2; note the reduction in bowel encompassed by the 57 Gy, 37 and 20 Gy isodoses in the DCR-VMAT plan. DVHs for all PTVs, bowel, rectum and bladder are presented in Figure 3(d), with OAR dose statistics presented in Table 6.

Considerable sparing of the bowel at clinically relevant dose levels was found with DCR-VMAT, with reductions in $\mathrm{V}_{45 G y}$ of $5 \%, \mathrm{~V}_{50 G y}$ of $9 \%, \mathrm{~V}_{55 G y}$ of $31 \%$ and $\mathrm{V}_{60 G y}$ of $45 \%$. Rectum and bladder doses were broadly comparable.

\section{Discussion}

Despite recent interest in rotational treatment techniques, there have been relatively few papers on the application of novel trajectories to VMAT. This paper presents preliminary results from applying Dijkstra's algorithm for calculating a least-cost path through a cost map to produce optimized non-coplanar VMAT trajectories, combining dynamic linac gantry and treatment couch rotation, that geometrically avoid organs at risk. To the authors' knowledge, this is the first paper to directly optimize DCRVMAT trajectories and the first to apply a graph search algorithm to the problem. The plan comparisons presented above show the impact that a heuristic technique can have, 
Table 6. Organ at risk dose statistics for the prostate and pelvic nodes case.

\begin{tabular}{|c|c|c|c|}
\hline \multirow[b]{2}{*}{ Organ at risk } & \multirow[b]{2}{*}{ Criterion } & \multicolumn{2}{|c|}{ Volume (\%) } \\
\hline & & VMAT & DCR-VMAT \\
\hline \multirow[t]{2}{*}{ Bladder } & $\mathrm{V}_{50 G y}$ & 84.4 & 83.8 \\
\hline & $\mathrm{V}_{60 G y}$ & 40.8 & 41.3 \\
\hline \multirow[t]{2}{*}{ Rectum } & $\mathrm{V}_{50 G y}$ & 47.1 & 44.9 \\
\hline & $\mathrm{V}_{60 G y}$ & 15.3 & 12.8 \\
\hline Left femoral head & $\mathrm{V}_{50 G y}$ & 4.1 & 3.0 \\
\hline \multirow[t]{3}{*}{ Right femoral head } & $\mathrm{V}_{50 G y}$ & 0.0 & 0.0 \\
\hline & & \multicolumn{2}{|c|}{ Volume (cc) } \\
\hline & & VMAT & DCR-VMAT \\
\hline \multirow[t]{2}{*}{ Bowel } & $\mathrm{V}_{50 G y}$ & 91.2 & 83.1 \\
\hline & $\mathrm{V}_{60 G y}$ & 38.4 & 21.0 \\
\hline
\end{tabular}

demonstrating the considerable clinical potential of trajectory optimization in critical organ sparing. In each clinical case, trajectory optimization successfully determined a DCR-VMAT trajectory which minimized the irradiation of specific OARs and improved DVHs over coplanar VMAT. More extensive site specific evaluations are required to fully quantify the expected benefit from clinical implementation of trajectory optimization.

Our trajectory optimization method compares favourably to existing DCR-VMAT literature. While it is difficult to compare the single right-sided breast case with the mixed cohorts reported by Shaitelman et al (2011) and Popescu et al (2013), Popescu et al reported differences for inner central lesions, with a decrease in contralateral breast maximum dose for DCR-VMAT of $32 \%$ compared with $23 \%$ in this paper, and increased mean dose to the ipsilateral breast of $13 \%$ (7\%). Yang et al (2011) compared coplanar VMAT with their DCR-VMAT optimization technique for a cohort of 10 brain patients. In that paper, average maximum doses were reduced to the brainstem by $1 \%$, optic chiasm by $2 \%$, optic nerves by $4 \%$, cochleae by $3 \%$, and lenses by $9 \%$, while globes increased by $1 \%$. These results compare favourably with those presented in Table 3. Further investigation of the potential benefits of optimized multiple partial-arc trajectories against single-arc DCR-VMAT is outside the scope of this paper but is of interest given the results of the pDCR-VMAT case presented. Bedford \& Warrington (2010) reported reductions in "low and intermediate" doses - approximately in the 0-35 Gy range of a 60 and 47 Gy simultaneous integrated boost 20 fraction prescription to bowel for five prostate and pelvic node plans with a bowel-sparing 'arbitrary' DCRVMAT trajectory but did not provide quantitative data. Bowel sparing in this paper's equivalent case was mostly in the 20-60 Gy range for a 74, 71 and 60 Gy SIB 37 fraction prescription. In all cases a more detailed comparison is required to contrast the different DCR-VMAT approaches. Integration of a more sophisticated collision detection algorithm, such as that described by Nioutsikou et al (2003), is also required to ensure the full range of clinically possible geometries is available in the optimization, 
and to minimize potential patient safety issues.

The trajectory optimization method presented is strictly a heuristic one, performing trajectory determination prior to beam aperture optimization. However, during VMAT delivery the beam aperture changes dynamically, irradiating or shielding organs at risk depending on the required radiation fluence for a specific source position. Ideally this should be reflected in the cost function for that geometry, which might result in an iterative process of aperture modification, cost map recalculation and subsequent reevaluation of the 'optimal' trajectory. To achieve this, trajectory optimization could be combined with an aperture optimization technique, such as direct aperture optimization (Shepherd et al 2002, Bedford \& Webb 2006). One barrier to combined trajectory and dose optimization is the large number of calculations required, suggesting the need for highly parallel computation, for example using a graphics processor unit (GPU), to maintain practical overall calculation times. However a recent paper by Ziegenhein et al (2013) demonstrated that, by using optimized code and hardware, central processor unit (CPU) dose recalculation could be significantly faster without the need for GPUs. Combining this approach with trajectory optimization would allow rapid cost map re-evaluation during a trajectory search, enabling a true, non-heuristic, trajectory optimization technique that was responsive to changes during clinical beam aperture optimization. Benchmarking against the heuristic approach presented in this paper, including evaluation of any trade-off in plan quality versus calculation time, could then be performed.

Prior to clinical implementation of any DCR-VMAT technique, the dosimetric impact of set-up and delivery errors must be fully quantified. Systematic misalignment of couch rotation in DCR-VMAT showed mixed results for the case tested, with minor differences in PTV and most OAR statistics but an almost $10 \%$ difference for a $2^{\circ}$ error in one OAR. A complete uncertainty analysis is outside the scope of this paper, however the combined effect of mechanical and dynamic delivery uncertainties (e.g. accuracy of couch and gantry position and speed), trajectory control point resolution, and the effect of dynamic couch rotation on intrafraction patient position all need consideration. The dosimetric effect of errors on DCR-VMAT plans is likely to be both site and trajectory dependent; a method of assessing DCR-VMAT robustness and incorporating robustness measures within trajectory optimization should be explored.

\section{Conclusion}

Trajectory optimization for DCR-VMAT is achievable by combining ray tracing with a graph search algorithm and produces DCR-VMAT trajectories that result in considerable reduction of organ at risk doses for plans otherwise comparable to conventional coplanar VMAT techniques. Further work on refining the trajectory optimization method presented to account for multiple OARs with different relative importance is planned. 


\section{Acknowledgments}

GS acknowledges the Clinical Radiotherapy Physics Group and the Neuro-Oncology Unit, Royal Marsden Hospital for supporting this work. We acknowledge support from the NIHR RM/ICR Biomedical Research Centre. Research at The Institute of Cancer Research is also supported by Cancer Research UK under Programme C46/A10588.

\section{References}

Bangert M 2011 New concepts for beam angle selection in IMRT treatment planning: From heuristics to combinatorial optimization. (PhD Thesis, University of Heidelberg) Available from: http://www.ub.uni-heidelberg.de/archiv/12272 Last accessed 10th July 2013

Bangert M, Oelfke U 2010 Spherical cluster analysis for beam angle optimization in intensity-modulated radiation therapy treatment planning. Phys. Med. Biol. 55 6023-37

Bangert M, Ziegenhein P, Oelfke U 2012 Characterizing the combinatorial beam angle selection problem. Phys. Med. Biol. 57 6707-23

Bangert M, Ziegenhein P, Oelfke U 2013 Comparison of beam angle selection strategies for intracranial IMRT. Med. Phys. 40011706

Bedford J L 2009 Treatment planning for volumetric modulated arc therapy.Med. Phys. 36 5128-38

Bedford J L 2013 Sinogram analysis of aperture optimization by iterative least-squares in volumetric modulated arc therapy. Phys. Med. Biol. 58 1235-50

Bedford J L, Warrington A P 2010 VMAT with an arbitrary trajectory. Proceedings of the XVIth ICCR

Bedford J L, Webb S 2006 Constrained segment shapes in direct-aperture optimization for step-andshoot IMRT. Med. Phys. 33 944-58

Bortfeld T, Schlegel W 1993 Optimization of beam orientations in radiation therapy: some theoretical considerations. Phys. Med. Biol. 38 291-304

Chen G T, Spelbring D R, Pelizzari C A, Balter J M, Myrianthopolous L C, Vijayakumar S, Halpern $\mathrm{H} 1992$ The use of beam's eye view volumetrics in the selection of non-coplanar radiation portals. Int. J. Radiat. Oncol. Biol. Phys. 23 153-63

Cormen T H, Leiserson C E, Rivest R L, Stein C 2009 Introduction to algorithms. Third edition. (Cambridge, MA: The MIT Press)

Dijkstra E W 1959 A note on two problems in connexion with graphs. Numerische mathematik $1269-71$

Donovan E M, Ciurlionis L, Fairfoul J, James H, Mayles H, Manktelow S, Raj S, Tsang Y, Tywman N, Yarnold J, Coles C 2011 Planning with intensity-modulated radiotherapy and tomotherapy to modulate dose across breast to reflect recurrence risk (IMPORT High trial). Int. J. Radiat. Oncol. Biol. Phys. 79 1064-72

Krayenbuehl J, Davis J B, Ciernik I F 2006 Dynamic intensity-modulated non-coplanar arc radiotherapy (INCA) for head and neck cancer. Radiother. Oncol. 81 151-7

McShan D L, Kessler M L, Fraass B A 1995 Advanced interactive planning techniques for conformal therapy: High level beam descriptions and volumetric mapping techniques. Int. J. Radiat. Oncol. Biol. Phys. 33 1061-72

Mizowaki T, Takayama K, Nagano K, Miyabe Y, Matsuo Y, Kaneko S, Kokuno M, Hiraoka M 2013 Feasibility evaluation of a new irradiation technique: three-dimensional unicursal irradiation with the Vero4DRT (MHI-TM2000). J. Radiat. Res. 54 330-6

Myrianthopoulos L C, Chen G T, Vijayakumar S, Halpern H J, Spelbring D R, Pelizzari C A 1992 Beam's eye view volumetrics: An aid in rapid treatment plan development and evaluation. Int. J. Radiat. Oncol. Biol. Phys. 23 367-75

Nioutsikou E, Bedford J L, Webb S 2003 Patient-specific planning for prevention of mechanical collisions during radiotherapy. Phys. Med. Biol. 48 N313-21

Otto K 2008 Volumetric modulated arc therapy: IMRT in a single gantry arc. Med. Phys. 35 310-7 
Podgorsak E B, Olivier A, Pla M, Lefebvre P-Y, Hazel J 1988 Dynamic stereotactic radiosurgery. Int. J. Radiat. Oncol. Biol. Phys. 14 115-26

Popescu C C, Beckham W A, Patenaude V V, Olivotto I A, Vlachaki M T 2013 Simultaneous couch and gantry dynamic arc rotation (CG-Darc) in the treatment of breast cancer with accelerated partial breast irradiation (APBI): a feasibility study. J. Appl. Clin. Med. Phys. 14 161-75

Pugachev A, Li J G, Boyer A L et al 2001 Role of beam orientation optimization in intensity-modulated radiation therapy. Int. J. Radiat. Oncol. Biol. Phys. 50 551-60

Pugachev A, Xing L 2001 Pseudo beam's-eye-view as applied to beam orientation selection in intensitymodulated radiation therapy. Int. J. Radiat. Oncol. Biol. Phys. 51 1361-70

Rowbottom C G, Nutting C M, Webb S 2001 Beam-orientation optimization of intensity-modulated radiotherapy: clinical application to parotid gland tumours. Radiother. Oncol. 59 163-72

Rowbottom C G, Webb S, Oldham M 1998 Improvements in prostate radiotherapy from the customization of beam directions. Med. Phys. 25 1171-9

Shaitelman S F, Kim L H, Yan D, Martinez A A, Vicini F A, Grills I S 2011 Continuous arc rotation of the couch therapy for the delivery of accelerated partial breast irradiation: a treatment planning analysis. Int. J. Radiat. Oncol. Biol. Phys. 80 771-8

Shepard D M, Earl M A, Li X A, Naqvi S, Yu C 2002 Direct aperture optimization: a turnkey solution for step-and-shoot IMRT. Med. Phys. 29 1007-18

Siddon R L 1981 Solution to treatment planning problems using coordinate transformations. Med. Phys. 8 766-74

Siddon R L 1985 Fast calculation of the exact radiological path for a three-dimensional CT array. Med. Phys. 12 252-5

Tree A C, Alexander E J, Van As N J, Dearnaley D P, Khoo V 2013 Biological dose escalation and hypofractionation: what is there to be gained and how will it best be done? Clin. Oncol. 25 483-98

Yang Y, Zhang P, Happersett L, Xiong J, Yang J, Chan M, Hunt M 2011 Choreographing couch and collimator in volumetric modulated arc therapy. Int. J. Radiat. Oncol. Biol. Phys. 80 1238-47

Yu C X 1999 Intensity-modulated arc therapy with dynamic multileaf collimation: an alternative to tomotherapy. Phys. Med. Biol. 40 1435-49

Yu C X, Tang G 2011 Intensity-modulated arc therapy: principles, technologies and clinical implementation. Phys. Med. Biol. 56 R31-54

Ziegenhein P, Kamerling C P, Bangert M, Kunkel J, Oelfke U 2013 Performance-optimized clinical IMRT planning on modern CPUs. Phys. Med. Biol. 58 3705-15 